\title{
Deciding key cycles for security protocols
}

\author{
Véronique Cortier ${ }^{1}$ and Eugen Zălinescu ${ }^{1}$ \\ Loria UMR 7503 \& INRIA Lorraine projet Cassis \& CNRS, France
}

\begin{abstract}
Many recent results are concerned with interpreting proofs of security done in symbolic models in the more detailed models of computational cryptography. In the case of symmetric encryption, these results stringently demand that no key cycle (e.g. $\{k\}_{k}$ ) can be produced during the execution of protocols. While security properties like secrecy or authentication have been proved decidable for many interesting classes of protocols, the automatic detection of key cycles has not been studied so far.

In this paper, we prove that deciding the existence of key-cycles is NP-complete for a bounded number of sessions. Next, we observe that the techniques that we use are of more general interest and apply them to reprove the decidability of a significant existing fragment of protocols with timestamps.
\end{abstract}

\section{Introduction}

Security protocols are small programs that aim at securing communications over a public network like Internet. The design of such protocols is difficult and error-prone; many attacks are discovered even several years after the publication of a protocol. Two distinct approaches for the rigorous design and analysis of cryptographic protocols have been pursued in the literature: the so-called Dolev-Yao, symbolic, or formal approach on the one hand and the cryptographic, computational, or concrete approach on the other hand. In the symbolic approach, messages are modeled as formal terms that the adversary can manipulate using a fixed set of operations. The main advantage of this approach is its relative simplicity which makes it amenable to automated analysis tools (see, e.g., $[9,4,22])$. In the cryptographic approach, messages are bit strings and the adversary is an arbitrary probabilistic polynomial-time Turing machine. While results in this model yield strong security guarantees, the proofs are often quite involved and only rarely suitable for automation (see, e.g., [17,7]).

Starting with the seminal work of Abadi and Rogaway [1], recent results investigate the possibility of bridging the gap between the two approaches. The goal is to obtain the best of both worlds: simple, automated security proofs that entail strong security guarantees. The approach usually consists in proving that the abstraction of cryptographic primitives made in the Dolev-Yao model is correct as soon as strong enough primitives are used in the implementation. For example, it has been shown [21] that the perfect encryption assumption is a sound abstraction for IND-CCA2, which corresponds to a well-established security level (a definition of this level of security can be found in [8]). The perfect encryption assumption intuitively states that encryption is a black-box that can be opened only when one has the inverse key. Otherwise, no information can be learned from a ciphertext about the underlying plaintext. 
However, it is not always sufficient to find the right cryptographic hypotheses. Formal models may need to be amended in order to be correct abstractions of the cryptographic models. This is in particular the case for symmetric encryption. For example, in [5], the authors consider extra-rules for the formal intruder in order to reflect the ability of a real intruder to choose its own keys in a particular manner.

A more widely used requirement is to control how keys can encrypt other keys. In a passive setting, soundness results $[1,19]$ require that no key cycles can be generated during the execution of a protocol. Key cycles are messages like enc $(k, k)$ or $\operatorname{enc}\left(k_{1}, k_{2}\right)$, enc $\left(k_{2}, k_{1}\right)$ where a key encrypts itself or more generally when the encryption relation between keys contains a cycle. Such key cycles have to be disallowed simply because usual security definitions for encryption schemes do not provide any guarantees when such key cycles occur. In the active setting, the typical hypotheses are even stronger. For instance, in $[5,18]$ the authors require that a key $k_{1}$ never encrypts a key $k_{2}$ generated before $k_{1}$.

Some authors circumvent the problem of key cycles by providing new security definitions for encryption that allow key cycles $[3,6]$. However, the standard security notions do not imply these new definitions and ad-hoc encryption schemes have to be constructed in order to satisfy the definitions. These constructions use the random oracle model which is provably non implementable. As a consequence, it is not known how to implement encryption schemes that satisfy the new definitions. In particular, none of the usual, implemented encryption schemes have been proved to satisfy the requirements.

Our main contribution is an NP-complete decision procedure for detecting the generation of key cycles during the execution of a protocol, in the presence of an intruder, for a bounded number of sessions. To the best of our knowledge, this problem has not been addressed before. We therefore provide a necessary component for automated tools used in proving strong, cryptographical security properties, using existing soundness results. Our result has been obtained following the classical approach of Rusinowitch-Turuani [24], revisited by Comon-Lundh [13, 15], where protocols are represented by constraint systems. Since this initial procedure is already implemented in Avispa [4] for deciding secrecy and authentication properties, we believe that our algorithm can be easily implemented since it can be adapted from the existing procedure.

Our second contribution is to provide a generic approach derived from $[13,15]$ to decide general security properties. Comon-Lundh showed that any constraint system can be transformed in (possibly several) much simpler constraint systems that are called solved forms. We show using (almost) the same transformation rules that, in order to verify a property on a constraint system, it is always sufficient to verify the property on the solved forms obtained after transformation. Compared to [13,15], the framework is slightly extended since we consider sorted terms, symmetric and asymmetric encryption, pairing and signatures. We use this approach to first prove NP-completeness of the key-cycle problem but also to show co-NP-completeness of secrecy for protocols with timestamps. We actually retrieve a significant fragment of the decidable class identified by Bozga et al [11]. We believe our result can lead more easily to an implementation since, again, we only need to adapt the procedure implemented in Avispa [4] while 
Bozga et al have designed a completely new decision procedure, which de facto has not been implemented.

The messages and the intruder capabilities are modeled in Section 2. In Section 3.1, we define constraint systems and show how they can be used to express protocol executions. In Section 3.2, we define security properties and the notion of satisfiability of constraint systems. In 3.3, we explain how the satisfiability problem of any security property can be reduced to the satisfiability of the same problem but on simpler constraint systems. We show in Section 4.1 how this approach can be used to obtain our main result of NP-completeness of the generation of key cycles and in Section 4.2 how it can be used to derive NP-completeness for protocols with timestamps. Some concluding remarks about further work can be found in Section 5.

\section{Messages and intruder capabilities}

\subsection{Syntax}

Cryptographic primitives are represented by function symbols. More specifically, we consider the signature $(\mathcal{S}, \mathcal{F})$ made of a set of sorts $\mathcal{S}=\left\{s, s_{1} \ldots\right\}$ and a set of symbols $\mathcal{F}=\{$ enc, enca, sign, \langle\rangle , pub, priv $\}$ together with arities of the form $\operatorname{ar}(f)=$ $s_{1} \times s_{2} \rightarrow s$ for the four first symbols and $\operatorname{ar}(f)=s \rightarrow s^{\prime}$ for the two last ones. The symbol \langle\rangle represents the pairing function. The terms enc $(m, k)$ and enca $(m, k)$ represent respectively the message $m$ encrypted with the symmetric (resp. asymmetric) key $k$. The term $\operatorname{sign}(m, k)$ represents the message $m$ signed by the key $k$. The terms $\operatorname{pub}(a)$ and $\operatorname{priv}(a)$ represent respectively the public and private keys of an agent $a$.

We fix an infinite set of names $\mathcal{N}=\{a, b \ldots\}$ and an infinite set of variables $\mathcal{X}=\{x, y \ldots\}$. We assume that names and variables are given with sorts. The set of terms of sort $s$ is defined inductively by

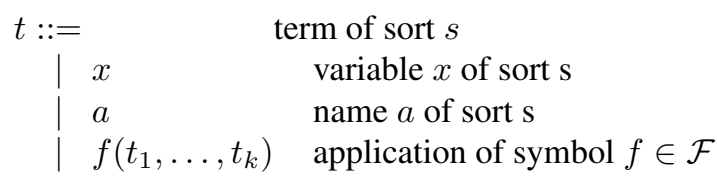

where for the last case, we further require that $t_{i}$ is a term of some sort $s_{i}$ and $\operatorname{ar}(f)=$ $s_{1} \times \ldots \times s_{k} \rightarrow s$. We assume a special sort Msg that subsumes all the other sorts and such that any term is of sort Msg.

As usual, we write $\mathcal{V}(t)$ for the set of variables occurring in $t$. A term is ground or closed if and only if it has no variables. The size of a term $t$, denoted $|t|$, is defined inductively as usual: $|t|=1$ if $t$ is a variable or a name and $t=1+\sum_{i=1}^{n}\left|t_{i}\right|$ if $t=f\left(t_{1}, \ldots, t_{n}\right)$ for $f \in \mathcal{F}$. If $T$ is a set of terms then $|T|$ denotes the sum of the sizes of its elements. We denote by $S t(t)$ the set of subterms of $t$.

Substitutions are written $\sigma=\left\{x_{1}=t_{1}, \ldots, x_{n}=t_{n}\right\}$ with $\operatorname{dom}(\sigma)=\left\{x_{1}, \ldots, x_{n}\right\}$. We only consider well-sorted substitutions, that is substitutions for which $x_{i}$ and $t_{i}$ have the same sort. $\sigma$ is closed if and only if all of the $t_{i}$ are closed. The application of a substitution $\sigma$ to a term $t$ is written $\sigma(t)=t \sigma$. 


$$
\begin{array}{cccc}
\frac{S \vdash x \quad S \vdash y}{S \vdash\langle x, y\rangle} \quad \frac{S \vdash x \quad S \vdash y}{S \vdash \operatorname{enc}(x, y)} & \frac{S \vdash x \quad S \vdash y}{S \vdash \operatorname{enca}(x, y)} & \frac{S \vdash x \quad S \vdash y}{S \vdash \operatorname{sign}(x, y)} \\
\frac{S \vdash\langle x, y\rangle}{S \vdash x} & \frac{S \vdash\langle x, y\rangle}{S \vdash y} & \frac{S \vdash \operatorname{enc}(x, y) \quad S \vdash y}{S \vdash x} \\
\frac{S \vdash \operatorname{enca}(x, \operatorname{priv}(y))}{S \vdash x} \quad S \vdash \operatorname{pub}(y) & \frac{S \vdash \operatorname{sign}(x, \operatorname{priv}(y))}{S \vdash x} \text { (optional) }
\end{array}
$$

Fig. 1. Intruder deduction system.

Sorts are mostly left unspecified in this paper. They can be used in applications to express that certain operators can be applied only to some restricted terms. For example, sorts can be used to require that messages are encrypted only by atomic keys.

\subsection{Intruder capabilities}

The ability of the intruder is modeled by a deduction system described in Figure 1 and corresponds to the usual Dolev-Yao rules. The first line describes the composition rules, the two last lines the decomposition rules. Intuitively, these deduction rules say that an intruder can compose messages by pairing, encrypting and signing messages provided he has the corresponding keys and conversely, it can decompose messages by projecting or decrypting provided it has the decryption keys. For signatures, the intruder is also able to verify whether a signature $\operatorname{sign}(m, k)$ and a message $m$ match (provided she has the verification key), but this does not give her any new message. That is why this capability is not represented in the deduction system. We also consider an optional rule $\frac{S \vdash \operatorname{sign}(x, \operatorname{priv}(y))}{S \vdash x}$ that expresses that an intruder can retrieve the whole message from its signature. This property may or may not hold depending on the signature scheme, and that is why this rule is optional. All our results hold in both cases (deduction relation $\vdash$ defined with or without this rule).

A term $u$ is deducible from a set of terms $S$, denoted by $S \vdash u$ if there exists a proof i.e. a tree such that the root is $S \vdash u$, the leaves are of the form $S \vdash v$ with $v \in S$ (axiom rule) and every intermediate node is an instance of one of the rules of the deduction system.

Example 1. The term $\left\langle k_{1}, k_{2}\right\rangle$ is deducible from the set $S_{1}=\left\{\operatorname{enc}\left(k_{1}, k_{2}\right), k_{2}\right\}$. A proof of $S_{1} \vdash\left\langle k_{1}, k_{2}\right\rangle$ is:

$$
\frac{S_{1} \vdash \operatorname{enc}\left(k_{1}, k_{2}\right) \quad S_{1} \vdash k_{2}}{\frac{S_{1} \vdash k_{1}}{S_{1} \vdash\left\langle k_{1}, k_{2}\right\rangle} S_{1} \vdash k_{2}}
$$




\section{Constraint systems and security properties}

Constraint systems are quite common (see e.g. $[15,24]$ ) in modeling security protocols. We recall here its formalism and show how it can be used to specify general security properties. Then we prove that any constraint system can be transformed into some more simplified constraint systems.

\subsection{Constraint systems}

Definition 1. A constraint system $C$ is a finite set of expressions $T_{i} \Vdash \sharp$ or $T_{i} \Vdash u_{i}$, where $T_{i}$ is a non empty set of terms, $t$ is a special symbol that represents an always deducible term, and $u_{i}$ is a term, $1 \leq i \leq n$, such that:

- $T_{i} \subseteq T_{i+1}$, for all $1 \leq i \leq n-1$;

- if $x \in \mathcal{V}\left(T_{i}\right)$ then $\exists j<i$ such that $T_{j}=\min \{T \mid T \Vdash u \in C, x \in \mathcal{V}(u)\}$ (for the inclusion relation) and $T_{j} \subsetneq T_{i}$.

The left-hand side (right-hand side) of a constraint $T \Vdash u$ is $T$ (respectively $u$ ). The lefthand side of a constraint system $C$, denoted by lhs $(C)$, is the maximal set of messages $T_{n}$. The right-hand side of a constraint system $C$, denoted by $r h s(C)$, is the set of right-hand sides of its constraints. $\mathcal{V}(C)$ denotes the set of variables occurring in $C . \perp$ denotes the unsatisfiable system.

The size of a constraint system is defined as $|C| \stackrel{\text { def }}{=}|\operatorname{lhs}(C)|+|r h s(C)|$.

A constraint system is denoted as a conjunction of expressions. The left-hand side of a constraint system $C$ usually represents the messages sent on the network.

Example 2. Consider the famous Needham-Schroeder asymmetric key authentication protocol [20] designed for mutual authentication.

$$
\begin{array}{ll}
A \rightarrow B: & \operatorname{enca}\left(\left\langle N_{A}, A\right\rangle, \operatorname{pub}(B)\right) \\
B \rightarrow A: & \operatorname{enca}\left(\left\langle N_{A}, N_{B}\right\rangle, \operatorname{pub}(A)\right) \\
A \rightarrow B: & \operatorname{enca}\left(N_{B}, \operatorname{pub}(B)\right)
\end{array}
$$

The agent $A$ sends to $B$ his name and a fresh nonce (a randomly generated value) encrypted with the public key of $B$. The agent $B$ answers by copying $A$ 's nonce and adds a fresh nonce $N_{B}$, encrypted by $A$ 's public key. The agent $A$ acknowledges by forwarding $B$ 's nonce encrypted by $B$ 's public key. We assume that a potential intruder has a complete control of the network: he may intercept, block and send new messages to arbitrary agents.

Let $S_{0}=\{a, b, i, \operatorname{pub}(a), \operatorname{pub}(b), \operatorname{pub}(i), \operatorname{priv}(i)\}$ be the initial knowledge of the intruder. The following constraint system $C_{1}$ models a scenario where $A$ starts a session with a corrupted agent $I$ (whose private key is known to the intruder) and $B$ is willing to answer to $A$. We consider this very simple scenario for simplicity but of course we could also consider $A$ talking to $B$ and $B$ responding to $I$ for example.

$$
\begin{gathered}
S_{1} \stackrel{\text { def }}{=} S_{0} \cup\left\{\operatorname{enca}\left(\left\langle n_{a}, a\right\rangle, \operatorname{pub}(i)\right)\right\} \Vdash \operatorname{enca}(\langle x, a\rangle, \operatorname{pub}(b)) \\
S_{2} \stackrel{\text { def }}{=} S_{1} \cup\left\{\operatorname{enca}\left(\left\langle x, n_{b}\right\rangle, \operatorname{pub}(a)\right)\right\} \Vdash \operatorname{enca}\left(\left\langle n_{a}, y\right\rangle, \operatorname{pub}(a)\right) \\
S_{3} \stackrel{\text { def }}{=} S_{2} \cup\{\operatorname{enca}(y, \operatorname{pub}(i))\} \Vdash \operatorname{enca}\left(n_{b}, \operatorname{pub}(b)\right)
\end{gathered}
$$


where $n_{a}$ and $n_{b}$ are names of sort Msg and $x$ and $y$ are variables of sort Msg. The set $S_{1}$ represents the messages known to the intruder once $A$ has contacted the corrupted agent $I$. Then the equations 1 and 2 can be read as follows: if a message of the form enca $(\langle x, a\rangle, \operatorname{pub}(b))$ can be sent on the network, then $B$ would answer to this message by enca $\left(\left\langle x, n_{b}\right\rangle, \operatorname{pub}(a)\right)$, which is added to $S_{1}$. Subsequently, if a message of the form enca $\left(\left\langle n_{a}, y\right\rangle, \operatorname{pub}(a)\right)$ can be sent on the network, then $A$ would answer by enca $(y, \operatorname{pub}(i))$ since $A$ believes she is talking to $I$. The run is successful if $B$ can finish his session by receiving the message enca $\left(n_{b}, \operatorname{pub}(b)\right)$. Then $B$ believes he has talked to $A$ while $A$ actually talked to $I$. If the protocol was secure, such a constraint system should not have a solution. The variables represent those parts of messages that are a priori unknown to the agents.

\subsection{Security properties}

A security property is modeled by a predicate on (lists of) terms. The terms represent some information about the execution of the protocol, for example the messages that are sent on the network.

Definition 2. A security property is a predicate on lists of messages. For a list $L$ we denote by $L_{s}$ the set of messages of the list $L$.

Let $C$ be a constraint system, $L$ a list of terms such that $\mathcal{V}\left(L_{s}\right) \subseteq \mathcal{V}(C)$ and $P$ a security property. A solution of $C$ for $P$ w.r.t. $L$ is a closed substitution $\theta$ such that $\forall T \Vdash u \in C, T \theta \vdash u \theta$ and $P(L \theta)$ holds. Every substitution satisfies $T \Vdash t$ and none satisfies $\perp$.

Example 3. If the predicate $P$ is simply the true predicate (which holds for any list of terms) and the only sort is Msg then we simply retrieve the usual constraint system deduction problem, which is known to be NP-complete [13, 25].

Example 4. Secrecy can be easily expressed by requiring that the secret data is not deducible from the messages sent on the network. We consider again the constraint system $C_{1}$ defined in Example 2. Let $L_{1}$ be a list of the messages in $\operatorname{lhs}\left(C_{1}\right)$. We define the predicate $P_{1}$ to hold on a list of messages if and only if $n_{b}$ is deducible from it.

$$
P_{1}(L)=\text { true iff } L_{s} \vdash n_{b}
$$

Then the substitution

$$
\sigma_{1}=\left\{x=n_{a}, y=n_{b}\right\}
$$

is a solution of $C_{1}$ for the property $P_{1}$ w.r.t. $L_{1}$ and corresponds to the attack found by G. Lowe [20]. Note that such a deduction-based property could be directly encoded in the constraint system by adding a constraint $T \Vdash n_{b}$ where $T=\operatorname{lh} s\left(C_{1}\right)$.

Example 5. Authentication can also be defined using a predicate $P_{2}$ on lists of messages. Consider again the constraint system $C_{1}$ defined in Example 2 where $S_{0}$ is replaced by $S_{0}^{\prime}=S_{0} \cup\left\{\operatorname{enca}\left(\left\langle n_{a}^{\prime}, a\right\rangle\right.\right.$, pub $\left.\left.(b)\right)\right\}$ : the agent $A$ also initiates a session with $B$. We can express that when the protocol is completed, the agent $B$ should have accepted only the nonce $n_{a}^{\prime}$ generated by $A$ for $B$. More precisely, we consider $L_{2}=[x]$ 


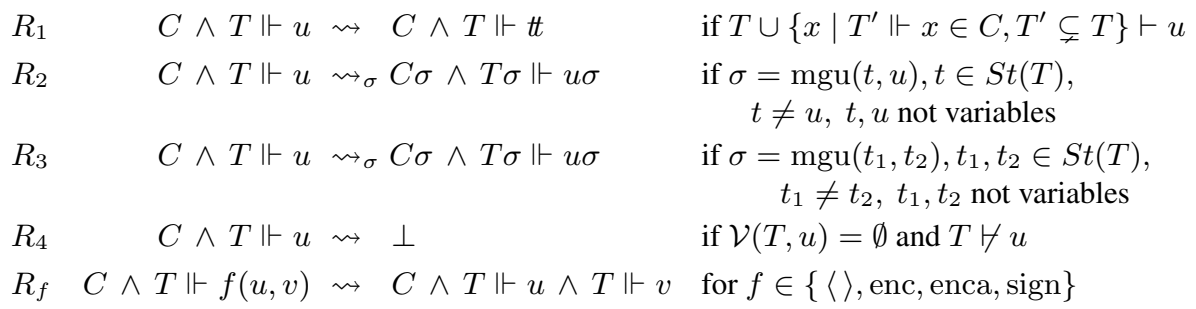

Fig. 2. Simplification rules.

representing the value received by $B$ for $n_{a}$. We define $P_{2}$ to hold on $L$ if and only if $L \neq\left[n_{a}\right]$. The substitution $\sigma_{1}$ defined in Example 4 is a solution of $C_{1}$ for the property $P_{2}$ w.r.t. $L_{2}$.

In Section 4, we provide other examples of predicates which encode time constraints or express that no key cycles are allowed.

\subsection{General approach}

Using some simplification rules, solving general constraint systems can be reduced to solving simpler constraint systems that we called solved. We say that a constraint system is solved if it is different from $\perp$ and each of its constraints are of the form $T \Vdash \sharp$ or $T \Vdash x$, where $x$ is a variable. This corresponds to the notion of solved form in [15].

Solved constraint systems with the single sort Msg are particularly simple in the case of the true predicate since they always have a solution, as noticed in [13]. Indeed, let $T_{1}$ be the smallest (w.r.t. inclusion) left hand side of a constraint. From the definition of a constraint system we have that $T_{1}$ is non empty and has no variables. Let $t \in T_{1}$. Then the substitution $\theta$ defined by $x \theta=t$ for every variable $x$ is a solution since $T \vdash x \theta=t$ for any constraint $T \Vdash x$ of the solved system.

The simplification rules we consider are defined in Figure 2. All the rules are in fact indexed by a substitution: when there is no index then the identity substitution is implicitly considered. We write $C \rightsquigarrow_{\sigma}^{n} C^{\prime}$ if there are $C_{1}, \ldots, C_{n}$ with $n \geq 1$, $C^{\prime}=C_{n}, C \rightsquigarrow \sigma_{1} C_{1} \rightsquigarrow \sigma_{2} \cdots \rightsquigarrow \sigma_{n} C_{n}$ and $\sigma=\sigma_{1} \sigma_{2} \ldots \sigma_{n}$. We write $C \rightsquigarrow{ }_{\sigma}^{*} C^{\prime}$ if $C{ }_{\sigma}^{n} C^{\prime}$ for some $n \geq 1$, or $C^{\prime}=C$ and $\sigma$ is the empty substitution.

The simplification rules are correct, complete and terminating in polynomial time.

Theorem 1. Let $C$ be a constraint system, $\theta$ a substitution, $P$ a security property and $L$ a list of messages such that $\mathcal{V}\left(L_{s}\right) \subseteq \mathcal{V}(C)$.

1. (Correctness) If $C \rightsquigarrow_{\sigma}^{*} C^{\prime}$ for some constraint system $C^{\prime}$ and some substitution $\sigma$ and if $\theta$ is a solution of $C^{\prime}$ for the property $P$ w.r.t. L $\sigma$ then $\sigma \theta$ is a solution of $C$ for the property $P$ w.r.t. $L$.

2. (Completeness) If $\theta$ is a solution of $C$ for the property $P$ w.r.t. L, then there exist a constraint system $C^{\prime}$ and substitutions $\sigma, \theta^{\prime}$ such that $\theta=\sigma \theta^{\prime}, C \rightsquigarrow_{\sigma}^{*} C^{\prime}$ and $\theta^{\prime}$ is a solution of $C^{\prime}$ for the property $P$ w.r.t. $L \sigma$. 
3. (Termination) If $C \rightsquigarrow_{\sigma}^{n} C^{\prime}$ for some constraint system $C^{\prime}$ and some substitution $\sigma$ then $n$ is polynomially bounded in the size of $C$.

The proof is a simple extension of the proof provided in [13] to sorted messages and general security properties. This last point simply relies on the fact that whenever $C \rightsquigarrow_{\sigma}^{*}$ $C^{\prime}$ then $L(\sigma \theta)=(L \sigma) \theta$ for any substitution $\theta$. All the proof details (mostly borrowed from [13]) can be found in the Appendix.

Corollary 1. Any property $P$ that can be decided in polynomial time on solved constraint systems can be decided in non-deterministic polynomial time on arbitrary constraint systems.

\section{Decidability of some specialized security properties}

Using the general approach presented in the previous section, verifying particular properties like the existence of key cycles or the conformation to an a priori given order relation on keys can be reduced to deciding these properties on solved constraint systems. We deduce a new decidability result, useful in models designed for proving cryptographic properties. This approach also allows us to retrieve a significant fragment of [11] for protocols with timestamps.

\subsection{Encryption cycles}

To show that formal models (like the one presented in this article) are sound with respect to cryptographical ones, the authors usually assume that no key cycle can be produced during the execution of a protocol or, even stronger, assume that the encrypts relation follows an a priori given order.

In this section we restrict our attention to key cycles and key order on symmetric keys since there are very few papers constraining the key relations in an asymmetric setting. We consider atomic keys for symmetric encryption since this is also the standard assumption in our setting (that is key cycles or key order definitions). More precisely, we assume a sort Key $\subset$ Msg and we assume that the sort of enc is Msg $\times$ Key $\rightarrow$ Msg. All the other symbols are of sort Msg $\times \cdots \times$ Msg $\rightarrow$ Msg. Hence only names and variables can be of sort Key.

Key cycles Many definitions of key cycles are available in the literature. They are defined by considering cycles in the encrypts relation between keys. But this relation differs from one article to another. For example, the early definition proposed by Abadi and Rogaway [2] says that $k$ encrypts $k^{\prime}$ as soon as there exists a term enc $(m, k)$ such that $k^{\prime}$ is a subterm of $m$. For example, both enc $(k, k)$ and enc $(\operatorname{enc}(a, k), k)$ contain key cycles. However, in the definition proposed by Laud [19], enc $(\operatorname{enc}(a, k), k)$ does not contain a key cycle since the relation " $k$ encrypts $k^{\prime \prime}$ " is restricted to keys $k^{\prime}$ that occur in plaintext. We consider the two variants for the notion of key cycles.

We write $s<_{s t} t$ if and only if $s$ is a subterm of $t$. We define recursively the least reflexive and transitive relation $\sqsubseteq$ satisfying: $s_{1} \sqsubseteq\left(s_{1}, s_{2}\right), s_{2} \sqsubseteq\left(s_{1}, s_{2}\right)$, and if $s \sqsubseteq t$ then $s \sqsubseteq \operatorname{enc}\left(t, t^{\prime}\right)$. Intuitively, $s \sqsubseteq t$ if $s$ is a subterm of $t$ that occurs at least once in a plaintext position. 
Definition 3. Let $\rho_{1}$ be a relation chosen in $\left\{<_{s t}\right.$, $€$. Let $S$ be a set of messages and $k, k^{\prime}$ two terms of sort Key. We say that $k$ encrypts $k^{\prime}$ in $S$ (denoted $k \rho_{e}^{S} k^{\prime}$ ) if there exist $m \in S$ and a term $m^{\prime}$ such that

$$
k^{\prime} \rho_{1} m^{\prime} \text { and } \operatorname{enc}\left(m^{\prime}, k\right) \sqsubseteq m .
$$

With $\rho_{1}=<_{s t}$, we retrieve the definition of Abadi and Rogaway. With $\rho_{1}=\sqsubseteq$ we retrieve the definition of Laud. For simplicity, we may write $\rho_{e}$ instead of $\rho_{e}^{S}$ if $S$ is clear from the context.

We say that a set of messages $S$ contains a key cycle if there is a cycle in the relation $\rho_{e}^{S}$. If $m$ is a message we denote by $\rho_{e}^{m}$ the relation $\rho_{e}^{\{m\}}$ and say that $m$ contains a cycle if $\{m\}$ contains a cycle.

Definition 4. Let $K$ be a set of names of sort Key. We define the predicate $P_{k c}^{K}$ as follows: $P_{k c}^{K}$ holds on a list of messages $L$ if and only if there is a message $m$ such that $L_{s} \vdash m$ and $m$ contains a key cycle $\left(k_{1}, \ldots, k_{n}\right)$, with $n \geq 1$ and $k_{i} \in K$ for all $1 \leq i \leq n$.

Key order In order to establish soundness of formal models in a symmetric encryption setting, the requirements on the encrypts relation can be even stronger, in particular in the case of an active intruder. In [5] and [18], the authors require that a key never encrypts a younger key. More precisely, the encrypts relation has to be compatible with the order in which the keys are generated. Hence we also want to check whether there exist executions of the protocol for which the encrypts relation is incompatible with a certain order on keys.

Definition 5. Let $\leq$ be a partial order on a set of names $K$ of sort Key. We define the predicate $P_{\leq}^{K}$ as follows: $P_{\leq}^{K}$ holds on a list of messages $L$ if and only if for any message $m$ such that $L_{s} \vdash m$ the encrypts relation $\rho_{e}^{m}$ is compatible with $\leq$, that is:

$$
k \rho_{e}^{m} k^{\prime} \Rightarrow k^{\prime} \not \leq k .
$$

For example, in $[5,18]$, the authors choose $\leq$ to be the order in which the keys are generated: $k \leq k^{\prime}$ if $k$ has been generated before $k^{\prime}$. We denote by $\bar{P}_{\leq}^{K}$ the negation of $P_{\leq}^{K}$. Indeed, an attack in this context is an execution such that the encrypts relation is incompatible with $\leq$, that is the predicate $\bar{P}_{\leq}^{K}$ holds.

We provide a characterization in the passive case for $P_{k c}^{K}$ and $\bar{P}_{\leq}^{K}$ to hold.

Proposition 1. Let $L$ be a list of messages, $K$ a set of names of sort Key and $\leq a$ partial order on $K$. The predicate $P_{k c}^{K}$ (resp. $\bar{P}_{\leq}^{K}$ ) holds if and only if

- there is $k \in K$ such that $k$ is deducible from $L_{s}$, that is $L_{s} \vdash k$, or

- $L_{s}$ contains a key cycle (resp. the encrypts relation on $L_{s}$ is not compatible with $\leq$ ).

Indeed if $k$ is deducible from $L_{s}$ then $\operatorname{enc}(k, k)$ is deducible from $L_{s}$. Hence there is a deducible message containing a key cycle and for which the encrypts relation is not compatible with the order $\leq$. If there are no deducible keys then the encrypts relation on any deducible message is included in the encrypts relation on $L_{s}$, hence the equivalences in the proposition. 
Decidability In what follows, a solution of $C$ for the true predicate w.r.t. an arbitrary list is said to be a partial solution to $C$. A key is a term of sort Key.

We show how to decide the existence of key cycles or the conformation to an order in polynomial time for solved constraint systems.

Proposition 2. Let $C$ be a solved constraint system and $L$ be a list of messages such that $\mathcal{V}\left(L_{s}\right) \subseteq \mathcal{V}(C)$, lhs $(C) \subseteq L_{s}$, and $L_{s} \cap$ Key $\neq \emptyset$. Indeed, the intruder should always know the messages sent on the network and some (dishonest) key. Let $K$ be a set of names of sort Key such that $L_{s} \theta \forall k$ for any $\theta$ partial solution of $C$ and for any $k \in K$. Let $\leq$ be a partial order on $K$.

- Deciding whether $C$ has a solution for $P_{k c}^{K}$ wrt $L$ can be done in $\mathcal{O}\left(|C|+|L|+|K|^{2}\right)$.

- Deciding whether $C$ has a solution for $\bar{P}_{\leq}^{K}$ wrt $L$ can be done in $\mathcal{O}\left(|C|+|L|+|K|^{2}\right)$.

The rest of the section is devoted to the proof of Proposition 2.

Since the keys of $K$ are not deducible from $L_{s} \theta$, for any $\theta$ partial solution of $C$, we know by Proposition 1 that it is sufficient to look at the encrypts relation only on $L_{s} \theta$ (and not on every deducible term).

Since $C$ is solved, any constraint of $C$ is of the form $T \Vdash x$ or $T \Vdash t$. For each variable $x$ of $C$ we denote $T_{x}=\min \{T \mid T \Vdash x \in C\}$. Let $t_{x}$ be the term obtained by pairing all terms of $T_{x}$ (in some arbitrary order). We construct the following substitution $\tau=\tau_{1} \ldots \tau_{q}$, where $q$ is the number of variables in $C$, and $\tau_{j}$ is defined inductively as follows:

- $\operatorname{dom}\left(\tau_{1}\right)=\left\{x_{1}\right\}$ and $x_{1} \tau_{1}=t_{x_{1}}$

- $\tau_{j+1}=\tau_{j} \cup\left\{{ }^{t_{x_{i}}} \tau_{j} / x_{i}\right\}$, where $i=\min \left\{i^{\prime} \mid x_{i^{\prime}} \notin \operatorname{dom}\left(\tau_{j}\right)\right\}$.

The construction is correct by the definition of a constraint system. It is clear that $\tau$ is a partial solution of $C$.

Consider $T_{L} \stackrel{\text { def }}{=} \max \left\{T_{x} \mid x \in \mathcal{V}\left(L_{s}\right)\right\}$. That is, $T_{L}$ is the maximal left hand side of a constraint of $C$ such that the right hand side variable appears in $L$. We construct the following directed graph $G=(K, A)$ as follows: if $k$ encrypts $k^{\prime}$ in $L_{s} \cup T_{L}$ then $\left(k, k^{\prime}\right) \in A$; and, if $k$ encrypts $x$ in $L_{s} \cup T_{L}$ (where $\left.x \in \mathcal{V}\left(L_{s} \cup T_{L}\right)\right)$ then $\left(k, k^{\prime}\right) \in A$ for all $k^{\prime} \rho_{1} x \tau$.

The graph captures exactly the encrypts relation induced by $\tau$ on $C$ and any possible encrypts relation is contained in the graph.

Lemma 1. Let $\theta$ be a partial solution of $C$ and $k, k^{\prime} \in K$ be two non deducible keys, that is $L_{s} \theta \forall k, k^{\prime}$. If $k \rho_{e}^{L_{s} \theta} k^{\prime}$, that is $k$ encrypts $k^{\prime}$ in $L_{s} \theta$ then $\left(k, k^{\prime}\right) \in A$. Conversely, if $\left(k, k^{\prime}\right) \in A$ then $k \rho_{e}^{L_{s} \tau} k^{\prime}$, that is $k$ encrypts $k^{\prime}$ in $L_{s} \tau$.

We deduce that deciding whether $C$ has a solution for $P_{k c}^{K}$ w.r.t. $L$ can be done simply by deciding whether the graph $G$ has cycles. Indeed, if there is a solution $\theta$ such that there is a cycle $\left(k_{1}, \ldots, k_{n}\right)$ in $L_{s} \theta$, that is, for each $i$ we have $k_{i} \rho_{e}^{L_{s} \theta} k_{i+1}$, then (by Lemma 1) $\left(k_{i}, k_{i+1}\right) \in A$ for each $i$, that is $\left(k_{1}, \ldots, k_{n}\right)$ is a cycle in the graph $G$. Conversely, if $\left(k_{1}, \ldots, k_{n}\right)$ is a cycle in $G$ then, again by Lemma $1, \tau$ is a solution of $C$ for $P_{k c}^{K}$ w.r.t. $L$. 
Deciding whether $C$ has a solution for $\bar{P}_{\leq}^{K}$ w.r.t. $L$ can be done by deciding whether the graph $G$ has the following property $P_{G}$ : there is $\left(k, k^{\prime}\right) \in A$ such that $k \leq k^{\prime}$. And indeed, if there is a solution $\theta$ such that $\bar{P}_{\leq}^{K}$ holds on $L \theta$, that is $P_{\leq}^{K}$ does not hold on $L \theta$, then there are $k, k^{\prime} \in K$ such that $k \rho_{e}^{L_{s} \theta} k^{\prime}$ and $k \leq k^{\prime}$. Hence $\left(k, k^{\prime}\right) \in A$ and $k \leq k^{\prime}$. That is the property $P_{G}$ on the graph $G$ holds. Conversely, if the property $P_{G}$ holds then there are $k, k^{\prime} \in K$ such that $k$ encrypts $k^{\prime}$ in $L \tau$ and $k \leq k^{\prime}$, that is $\tau$ is a solution of $C$ for $\bar{P}_{\leq}^{K}$ w.r.t. $L$.

The graph can be constructed in $\mathcal{O}\left(|C|+\left|L_{s}\right|+|K|^{2}\right)$. Testing for cycles and verifying property $P_{G}$ can be simply done by traversing the graph in $\mathcal{O}\left(|K|^{2}\right)$.

NP-completeness Consider a constraint system $C$, a set $K$ of names of sort Key and a list of messages $L$ such that $\mathcal{V}\left(L_{s}\right) \subseteq \mathcal{V}(C)$, lhs $(C) \subseteq L_{s}$ and $L_{s} \cap \mathrm{Key} \neq \emptyset$. We want to decide the existence of a solution of $C$ for $P_{k c}^{K}$ (resp. $P_{\leq}^{K}$ ) w.r.t. $L$. By Proposition 1, there is a solution if and only if

1. either there exists $k \in K$ such that there exists a partial solution to $C_{k} \stackrel{\text { def }}{=} C \wedge$ $L_{s} \Vdash k$,

2. or no key from $k \in K$ is deducible (that is $L_{s} \theta \nvdash k$, for all $\theta$ partial solution of $C$ ) and $C$ has a solution for $P_{k c}^{K}$ (resp. $P_{\leq}^{K}$ ) w.r.t. $L$.

We guess whether we are in case 1 or 2 and in case 1 we also guess which key is deducible. In the first case, we check whether $C_{k}$ has a partial solution in non-deterministic polynomial time using Corollary 1 . In the second case, we check whether $C$ has a solution for $P_{k c}^{K}$ (resp. $P_{\leq}^{K}$ ) w.r.t. $L$ using Theorem 1 and Proposition 2.

NP-hardness is obtained by adapting the construction for NP-hardness provided in [25]. More precisely, we consider the reduction of the 3SAT problem to our problem. For any 3SAT boolean formula we construct a protocol such that the intruder can deduce a key cycle if and only if the formula is satisfiable. The construction is the same as in [25] (pages 15 and 16) except that, in the last rule, the participant responds with the term enc $(k, k)$, for some fresh key $k$ (initially secret), instead of Secret. Then it is easy to see that the only way to produce a key cycle on a secret key is to play this last rule which is equivalent, using [25], to the satisfiability of the corresponding 3SAT formula.

\subsection{Timestamps}

For modeling timestamps, we introduce a new sort Time $\subseteq$ Msg for time and we assume an infinite number of names of sort Time, represented by rational numbers or integers. We assume that the only two sorts are Time and Msg. Any value of time should be known to an intruder, that is why we add to the deduction system the rule $\overline{S \vdash a}$ for any name $a$ of sort Time. All the previous results can be easily extended to such a deduction system since ground deducibility $(S \stackrel{?}{\vdash} u$ ) remains decidable in polynomial time.

To express relations between timestamps, we use timed constraints. An integer timed constraint or a rational timed constraint $T$ is a conjunction of formulas of the 
form

$$
\sum_{i=1}^{k} \alpha_{i} x_{i} \ltimes \beta
$$

where the $\alpha_{i}$ and $\beta$ are rational numbers, $\ltimes \in\{<, \leq\}$, and the $x_{i}$ are variables of sort Time. A solution of a rational (resp. integer) timed constraint $T$ is a closed substitution $\sigma=\left\{x_{1}=c_{1}, \ldots, x_{k}=c_{k}\right\}$, where the $c_{i}$ are rationals (resp. integers), that satisfies the constraint.

Timed constraints between the variables of sort Time are expressed through satisfiability of security properties.

Definition 6. A predicate $P$ is a timed property if $P$ is generated by some (rational or integer) timed constraint $T$, that is if $T$ has variables $x_{1}, \ldots, x_{k}$ then for any list $L$ of messages $P(L)$ holds if and only if

- $L$ contains exactly $k$ messages $t_{1}, \ldots, t_{k}$ of sort Time that appear in this order in the list

- and $T\left(t_{1}, \ldots, t_{k}\right)$ is true.

Such timed properties are typically used to say that a timestamp $x_{1}$ must be fresher than a timestamp $x_{2}\left(x_{1} \geq x_{2}\right)$ or that $x_{1}$ must be at least 30 seconds fresher than $x_{2}$ $\left(x_{1} \geq x_{2}+30\right)$.

Example 6. We consider the Wide Mouthed Frog Protocol [12].

$$
\begin{aligned}
& A \rightarrow S: A, \operatorname{enc}\left(\left\langle T_{a}, B, K_{a b}\right\rangle, K_{a s}\right) \\
& S \rightarrow B: \operatorname{enc}\left(\left\langle T_{s}, A, K_{a b}\right\rangle, K_{b s}\right)
\end{aligned}
$$

$A$ sends to a server $S$ a fresh key $K_{a b}$ intended for $B$. If the timestamp $T_{a}$ is fresh enough, the server answers by forwarding the key to $B$, adding its own timestamps. $B$ simply checks whether this timestamp is older than any other message he has received from $S$. As explained in [12], this protocol is flawed because an attacker can use the server to keep a session alive as long as he wants by replaying the answer of the server.

This protocol can be modeled by the following constraint system:

$$
\begin{gathered}
S_{1} \stackrel{\text { def }}{=}\left\{a, b,\left\langle a, \operatorname{enc}\left(\left\langle 0, b, k_{a b}\right\rangle, k_{a s}\right)\right\rangle\right\} \Vdash\left\langle a, \operatorname{enc}\left(\left\langle x_{t_{1}}, b, y_{1}\right\rangle, k_{a s}\right)\right\rangle, x_{t_{2}} \\
\left.S_{2} \stackrel{\text { def }}{=} S_{1} \cup\left\{\operatorname{enc}\left(\left\langle x_{t_{2}}, a, y_{1}\right\rangle, k_{b s}\right)\right\} \Vdash \operatorname{enc}\left(\left\langle x_{t_{3}}, a, y_{2}\right\rangle, k_{b s}\right)\right\rangle, x_{t_{4}} \\
\left.S_{3} \stackrel{\text { def }}{=} S_{2} \cup\left\{\operatorname{enc}\left(\left\langle x_{t_{4}}, b, y_{2}\right\rangle, k_{a s}\right)\right\} \Vdash \operatorname{enc}\left(\left\langle x_{t_{5}}, b, y_{3}\right\rangle, k_{a s}\right)\right\rangle, x_{t_{6}} \\
\left.S_{4} \stackrel{\text { def }}{=} S_{3} \cup\left\{\operatorname{enc}\left(\left\langle x_{t_{6}}, a, y_{3}\right\rangle, k_{b s}\right)\right\} \Vdash \operatorname{enc}\left(\left\langle x_{t_{7}}, b, k_{a b}\right\rangle, k_{b s}\right)\right\rangle
\end{gathered}
$$

where $y_{1}, y_{2}, y_{3}$ are variables of sort Msg and $x_{t_{1}}, \ldots, x_{t_{7}}$ are variables of sort Time. We add explicitly the timestamps emitted by the agents on the right hand side of the constraints (that is in the messages expected by the participants) since the intruder can schedule the message transmission whenever he wants.

Initially, the intruder simply knows the names of the agents and $A$ 's message at time 0 . Then $S$ answers alternatively to requests from $A$ and $B$. Since the intruder controls the network, the messages can be scheduled as fast as the intruder needs it. The server $S$ should not answer if $A$ 's timestamp is too old (let's say older than 30 seconds) 
thus $S$ 's timestamp cannot be too much delayed (no more than 30 seconds). This means that we should have $x_{t_{2}} \leq x_{t_{1}}+30$. Similarly, we should have $x_{t_{4}} \leq x_{t_{3}}+30$ and $x_{t_{6}} \leq x_{t_{5}}+30$. The last rule corresponds to $B$ 's reception. In this scenario, $B$ does not perform any check on the timestamp since it is the first message he receives.

We say that there is an attack if there is a solution to the constraint system that satisfies the previously mentioned time constraints and such that the timestamp received by $B$ is too fresh to come from $A: x_{t_{7}} \geq 90$. Formally, we consider the timed property generated by the following timed constraint

$$
x_{t_{2}} \leq x_{t_{1}}+30 \wedge x_{t_{4}} \leq x_{t_{3}}+30 \wedge x_{t_{6}} \leq x_{t_{5}}+30 \wedge x_{t_{7}} \geq 90
$$

Then the substitution corresponding to the attack is

$$
\sigma=\left\{y_{1}=y_{2}=y_{3}=y_{4}=k_{a b}, x_{t_{1}}=0, x_{t_{2}}=x_{t_{3}}=30, x_{t_{4}}=x_{t_{5}}=60, x_{t_{6}}=x_{t_{7}}=90\right\} \text {. }
$$

Proposition 3. Any timed property can be decided in non-deterministic polynomial time on solved constraints.

Proof (sketch). Let $C$ be a solved constraint, $P$ a timed property and $T$ a timed constraint generating $P$. Let $x_{1}, \ldots, x_{n}$ be the variables of sort Msg in $C$ and $y_{1}, \ldots, y_{k}$ the variables of sort Time in $C$. Clearly, any substitution $\sigma$ of the form $\sigma\left(x_{i}\right)=u_{i}$ where $u_{i} \in S_{i}$ for some $S_{i} \Vdash x_{i} \in C$ and $\sigma\left(y_{i}\right)=t_{i}$ for $t_{i}$ any constant of sort Time is a solution of $C$ for the true property. Let $\sigma^{\prime}$ be a the restriction of $\sigma$ to the timed variables $y_{1}, \ldots, y_{k}$.

Clearly, $\sigma$ is a solution of $C$ for $P$ if and only if $\sigma^{\prime}$ is a solution to $T$. Thus there exists a solution of $C$ for $P$ if and only if $T$ is satisfiable. The satisfiability of $T$ is solved by usual linear programming [26]. It is polynomial in the case of rational timed constraints and it is NP-complete in the case of integer timed constraints, thus the result.

NP-completeness We deduce by combining Theorem 1 and Proposition 2 that the problem of deciding timed properties on arbitrary constraint systems is in NP.

NP-hardness directly follows from the NP-hardness of constraint system solving by considering a predicate corresponding to an empty timed constraint.

\section{Further work}

We have shown how the generic approach we have derived from [13, 15, 24] can be used to retrieve two NP-completeness results. The first one enables us to detect key cycles while the second one enables us to solve constraint systems with timed constraints. In both cases, we had to provide a decision procedure only for a simple class of constraint systems. Since the constraint-based approach [13, 15, 24] has already been implemented in Avispa [4], we plan, using our results, to adapt this implementation to the case of key cycles and timestamps.

More generally, taking advantage of our generic approach, we would like to explore how decision procedures of distinct security properties can be combined on solved constraint systems. In addition, in our two cases, decidability on solved constraints systems 
was quite simple. It would be interesting to understand which classes of properties can be decided in the same manner.

Regarding key cycles, our approach is valid for a bounded number of sessions only. Secrecy is undecidable in general [16] for an unbounded number of sessions. Such an undecidability result could be easily adapted to the problem of detecting key cycles. Several decidable fragments have been designed [23, 14, 10,27] for secrecy and an unbounded number of sessions. We plan to investigate how such fragments could be used to decide key cycles.

Acknowledgment. We are particularly grateful to Michaël Rusinowitch and Bogdan Warinschi for their very helpful suggestions.

\section{References}

1. M. Abadi and P. Rogaway. Reconciling two views of cryptography. In Proc. of the International Conference on Theoretical Computer Science (IFIP TCS'00), volume 1872 of LNCS, pages 3-22, August 2000.

2. M. Abadi and Ph. Rogaway. Reconciling two views of cryptography (the computational soundness of formal encryption). Journal of Cryptology, 2:103-127, 2002.

3. Pedro Adão, Gergei Bana, Jonathan Herzog, and Andre Scedrov. Soundness of formal encryption in the presence of key-cycles. In Proc. 10th European Symposium on Research in Computer Security (ESORICS'05), volume 3679 of LNCS, pages 374-396, 2005.

4. A. Armando, D. Basin, Y. Boichut, Y. Chevalier, L. Compagna, J. Cuellar, P. Hankes Drielsma, P.C. Héam, O. Kouchnarenko, J. Mantovani, S. Mödersheim, D. von Oheimb, M. Rusinowitch, J. Santiago, M. Turuani, L. Viganò, and L. Vigneron. The Avispa tool for the automated validation of internet security protocols and applications. In Proc. of Computer Aided Verification (CAV'05), volume 3576 of LNCS, 2005.

5. M. Backes and B. Pfitzmann. Symmetric encryption in a simulatable Dolev-Yao style cryptographic library. In Proc. 17th IEEE Computer Science Foundations Workshop (CSFW'04), pages 204-218, 2004.

6. Michael Backes, Birgit Pfitzmann, and Andre Scedrov. Key-dependent message security under active attacks. Cryptology ePrint Archive, Report 2005/421, 2005. http://eprint.iacr.org/.

7. M. Bellare and P. Rogaway. Entity authentication and key distribution. In Advances in Cryptology - Crypto '93, 13th Annual International Cryptology Conference, volume 773 of LNCS, pages 232-249, 1993.

8. Mihir Bellare, Anand Desai, David Pointcheval, and Phillip Rogaway. Relations among notions of security for public-key encryption schemes. In Advances in Cryptology - Proceedings of CRYPTO '98, volume 1462 of $L N C S$, pages 26-45, Santa Barbara, California, USA, August 1998. Springer-Verlag.

9. Bruno Blanchet. An efficient cryptographic protocol verifier based on Prolog rules. In Proc. 14th IEEE Computer Security Foundations Workshop (CSFW'01), pages 82-96, 2001.

10. Bruno Blanchet and Andreas Podelski. Verification of cryptographic protocols: Tagging enforces termination. In Andrew Gordon, editor, Foundations of Software Science and Computation Structures (FoSSaCS'03), volume 2620 of LNCS, April 2003.

11. L. Bozga, C. Ene, and Y. Lakhnech. A symbolic decision procedure for cryptographic protocols with time stamps. In Proc. 15th International Conference on Concurrency Theory (CONCUR'04), LNCS, pages 177-192, London, England, 2004. Springer-Verlag.

12. J. Clark and J. Jacob. A survey of authentication protocol literature. Available at http://www.cs.york.ac.uk/ jac/ papers/drareviewps.ps, 1997. 
13. H. Comon-Lundh. Résolution de contraintes et recherche d'attaques pour un nombre borné

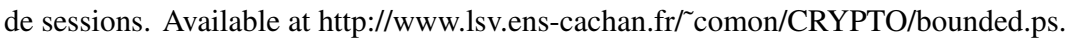

14. H. Comon-Lundh and V. Cortier. New decidability results for fragments of first-order logic and application to cryptographic protocols. In Proc. of the 14th Int. Conf. on Rewriting Techniques and Applications (RTA'2003), volume 2706 of LNCS, pages 148-164. SpringerVerlag, June 2003.

15. H. Comon-Lundh and V. Shmatikov. Intruder deductions, constraint solving and insecurity decision in presence of exclusive or. In Proc. of 18th Annual IEEE Symposium on Logic in Computer Science (LICS '03), pages 271-280, 2003.

16. N. Durgin, P. Lincoln, J. Mitchell, and A. Scedrov. Undecidability of bounded security protocols. In Proc. of the Workshop on Formal Methods and Security Protocols, 1999.

17. Shafi Goldwasser and Silvio Micali. Probabilistic encryption. Journal of Computer and System Sciences, 28:270-299, 1984.

18. R. Janvier, Y. Lakhnech, and L. Mazare. (de)compositions of cryptographic schemes and their applications to protocols. Cryptology ePrint Archive, Report 2005/020, 2005. http://eprint.iacr.org/.

19. P. Laud. Encryption cycles and two views of cryptography. In Nordic Workshop on Secure IT Systems (NORDSEC'02), 2002.

20. G. Lowe. Breaking and fixing the Needham-Schroeder public-key protocol using FDR. In Tools and Algorithms for the Construction and Analysis of Systems (TACAS'96), volume 1055 of LNCS, pages 147-166. Springer-Verlag, March 1996.

21. Daniele Micciancio and Bogdan Warinschi. Soundness of formal encryption in the presence of active adversaries. In Proc. 1st Theory of Cryptography Conference (TCC'04), volume 2951 of $L N C S$, pages 133-151, 2004.

22. J. K. Millen and V. Shmatikov. Constraint solving for bounded-process cryptographic protocol analysis. In Proc. 8th ACM Conference on Computer and Communications Security (CCS'01), pages 166-175, 2001.

23. R. Ramanujam and S.P.Suresh. Tagging makes secrecy decidable for unbounded nonces as well. In Proc. of the 23rd Conference on Foundations of Software Technology and Theoretical Computer Science (FSTTCS'03), Mumbai, 2003.

24. M. Rusinowitch and M. Turuani. Protocol insecurity with finite number of sessions is NPcomplete. In Proc. of the 14th Computer Security Foundations Workshop (CSFW'01), pages 174-190. IEEE Computer Society Press, 2001.

25. M. Rusinowitch and M. Turuani. Protocol insecurity with finite number of sessions and composed keys is NP-complete. Theoretical Computer Science, 299:451-475, 2003.

26. A. Schrijver. Theory of Linear and Integer Programming. Wiley, 1998.

27. Kumar Neeraj Verma, Helmut Seidl, and Thomas Schwentick. On the complexity of equational horn clauses. In Proc. of the 22th International Conference on Automated Deduction (CADE 2005), Lecture Notes in Computer Science, pages 337-352. Springer-Verlag, 2005. 


\section{A Properties of the simplification rules}

We prove in this section Theorem 1, that is the correctness, completeness and termination of the simplification rules (in subsections A.1, A.3 and A.2 respectively).

In what follows we call the deduction rules of Figure 1 pairing, symmetric and asymmetric encryption, signature and, first and second projection, symmetric and asymmetric decryption respectively.

Let $C$ be a constraint system. For any $T \Vdash u$ and $y \in \mathcal{V}(T)$, we define $T_{y}=$ $\min \left\{T^{\prime \prime} \mid T^{\prime \prime} \Vdash u^{\prime \prime}, y \in \mathcal{V}\left(u^{\prime \prime}\right)\right\}$.

\section{A.1 Correctness}

Lemma 2. The simplification rules transform a constraint system into a constraint system.

Proof. Suppose that $C$ is a constraint system, $C=\bigwedge_{i} T_{i} \Vdash u_{i}$ and $C \rightsquigarrow_{\sigma} C^{\prime}$. Since $T_{i} \subseteq T_{i+1}$ implies $T_{i} \sigma \subseteq T_{i+1} \sigma$ we have that $C^{\prime}$ meets point 1 of the definition of constraint systems.

We show that that $C^{\prime}$ also meets point 2 of the definition of constraint systems. Let $T^{\prime} \Vdash u^{\prime} \in C^{\prime}$ and $x \in \mathcal{V}\left(T^{\prime}\right)$. We have to prove that $T_{x}^{\prime}$ exists and $T_{x}^{\prime} \subsetneq T^{\prime}$. We consider which simplification rule was applied.

- If rule $R_{1}$ was applied, eliminating the constraint $T \Vdash u$ from $C$, then $T^{\prime} \Vdash u^{\prime} \in C$ and

$$
\begin{aligned}
T_{x}^{\prime} & =\min \left\{T^{\prime \prime} \mid T^{\prime \prime} \Vdash u^{\prime \prime} \in C^{\prime}, x \in \mathcal{V}\left(u^{\prime \prime}\right)\right\} \\
& =\min \left\{T^{\prime \prime} \mid T^{\prime \prime} \Vdash u^{\prime \prime} \in C \backslash\{T \Vdash u\}, x \in \mathcal{V}\left(u^{\prime \prime}\right)\right\} .
\end{aligned}
$$

If $\min \left\{T^{\prime \prime} \mid T^{\prime \prime} \Vdash u^{\prime \prime} \in C \backslash\{T \Vdash u\}, x \in \mathcal{V}\left(u^{\prime \prime}\right)\right\}=\min \left\{T^{\prime \prime} \mid T^{\prime \prime} \Vdash u^{\prime \prime} \in C\right.$, $\left.x \in \mathcal{V}\left(u^{\prime \prime}\right)\right\}$ then the property is clearly satisfied (as it is satisfied by $T^{\prime} \Vdash u^{\prime}$ in $C$ ). Otherwise, we have that $x \in \mathcal{V}(u)$ and $T=\min \left\{T^{\prime \prime} \mid T^{\prime \prime} \Vdash u^{\prime \prime} \in C\right.$, $\left.x \in \mathcal{V}\left(u^{\prime \prime}\right)\right\}$. By minimality of $T$ it follows that $x \notin \mathcal{V}(T)$ and $x \notin\left\{y \mid T^{\prime \prime} \Vdash y \in\right.$ $\left.C, T^{\prime \prime} \subsetneq T\right\}$. Since $x \in \mathcal{V}(u)$, we have that $T \cup\left\{y \mid T^{\prime \prime} \Vdash y \in C, T^{\prime \prime} \subsetneq T\right\} \forall u$ which contradicts the applicability of rule $R_{1}$.

- If rules $R_{2}$ or $R_{3}$ were applied then there is an one-to-one relation between the constraints of $C$ and $C^{\prime}$ respectively. Consider $T \Vdash u \in C$ such that $T \sigma=T^{\prime}$ and $u \sigma=u^{\prime}$.

If $x$ is not introduced by $\sigma$ then $x \in \mathcal{V}(T)$. Then $T_{x}$ exists and $T_{x} \subsetneq T$. Then $T_{x} \sigma \subseteq T \sigma$. If $T_{x} \sigma=T \sigma$ then $x \in \mathcal{V}\left(T_{x}\right)$ which contradicts the minimality of $T_{x}$. Thus $T_{x} \sigma \subsetneq T \sigma$. We also have that $\left\{T^{\prime \prime} \sigma \mid T^{\prime \prime} \Vdash u^{\prime \prime} \in C, x \in \mathcal{V}\left(u^{\prime \prime}\right)\right\} \subseteq$ $\left\{T^{\prime \prime} \sigma \mid T^{\prime \prime} \sigma \Vdash u^{\prime \prime} \sigma \in C^{\prime}, x \in \mathcal{V}\left(u^{\prime \prime} \sigma\right)\right\}$, since for any term $u^{\prime \prime}$, if $x \in \mathcal{V}\left(u^{\prime \prime}\right)$ then $x \in \mathcal{V}\left(u^{\prime \prime} \sigma\right)$. It follows that $T_{x}^{\prime}$ exists and $T_{x}^{\prime} \subseteq T_{x} \sigma$. Hence $T_{x}^{\prime} \subsetneq T^{\prime}$.

Otherwise, suppose $x$ is introduced by $\sigma$, that is $\exists y \in \mathcal{V}(T)$ such that $x \in \mathcal{V}(y \sigma)$. Then $T_{y}$ exists and $T_{y} \subsetneq T$. We choose $y$ such that $T_{y}$ is minimal with respect to the inclusion relation. We have that $T_{x}^{\prime} \subseteq \min \left\{T^{\prime \prime} \sigma \mid T^{\prime \prime} \Vdash u^{\prime \prime} \in C, z \in \mathcal{V}\left(u^{\prime \prime}\right), x \in\right.$ $\mathcal{V}(z \sigma)\} \subseteq T_{y} \sigma$. Again $T_{y} \subsetneq T$ implies $T_{y} \sigma \subsetneq T \sigma$ otherwise $T_{y} \sigma=T \sigma$ implies that there exists $z \in \mathcal{V}\left(T_{y}\right)$ such that $x \in \mathcal{V}(z \sigma)$. We have $z \neq y$ by minimality of $T_{y}$. Thus there exists $T_{z} \subsetneq T_{y}$, which contradicts the minimality of $y$. Hence $T_{x}^{\prime}$ exists and $T_{x}^{\prime} \subsetneq T^{\prime}$. 
- If rule $R_{4}$ was applied then the obtained result is a constraint system by definition.

- If rule $R_{f}$ was applied then the property is preserved since if $x \in u^{\prime \prime}$, for some term $u^{\prime \prime}$ such that $T^{\prime \prime} \Vdash u^{\prime \prime} \in C^{\prime}$ then there is a term $v$ such that $T^{\prime \prime} \Vdash v \in C$.

Lemma 3 (correctness). If $C \rightsquigarrow_{\sigma} C^{\prime}$ then for every solution $\tau$ of $C^{\prime}$ for the property $P$ w.r.t $L \sigma, \sigma \tau$ is a solution of $C$ for the property $P$ w.r.t. $L$.

Proof. If the applied rule was $R_{1}$ then we have to prove that $T \tau \vdash u \tau$, where $T \Vdash u$ is the constraint replaced by $T \Vdash t$. We know that $T \cup\left\{x \mid T^{\prime} \Vdash x \in C, T^{\prime} \subsetneq T\right\} \vdash u$. It follows that $T \tau \cup\left\{x \tau \mid T^{\prime} \Vdash x \in C, T^{\prime} \subsetneq T\right\} \vdash u \tau$. And since for any $T^{\prime} \Vdash x \in$ $C, T^{\prime} \subsetneq T$, we have that $T^{\prime} \tau \vdash x \tau$, and hence $T \tau \vdash x \tau$, we obtain that $T \tau \vdash u \tau$.

Suppose that the rule applied in order to obtain $C^{\prime}$ was $R_{2}$ or $R_{3}$. Then we have for each constraint $T \Vdash u$ of $C$ that $(T \sigma) \tau \vdash(u \sigma) \tau$, that is, $T(\sigma \tau) \vdash u(\sigma \tau)$. If the rule was $R_{f}$ then we obtain that $T \tau \vdash f(u, v) \tau$ from $T \tau \vdash u \tau$ and $T \tau \vdash v \tau$ by applying the corresponding rule (e.g. encryption if $f=$ enc). Finally, rule $R_{4}$ couldn't have been applied.

We deduce that $\sigma \tau$ satisfies $C$. Moreover, since $\tau$ is solution of $C^{\prime}$ for the property $P$ w.r.t $L \sigma$, it means that $P((L \sigma) \tau)$ holds, that it $P(L(\sigma \tau))$ thus $\sigma \tau$ is solution of $C$ for the property $P$ w.r.t $L$.

\section{A.2 Termination and solved forms}

Lemma 4 (termination). If $C \rightsquigarrow_{\sigma}^{n} C^{\prime}$ for some constraint system $C^{\prime}$ and some substitution $\sigma$ then $n$ is polynomially bounded in the size of $C$.

Proof. To show termination, we assume that terms are represented by DAGs (directed acyclic graphs), which allow terms to share identical subterms. The reader is refereed to [25] for a definition of DAG represented terms. Consider the following measure associated to a constraint system $C, I(C)=(|\mathcal{V}(C)|,|C|)$, where the pairs are ordered lexicographically. No rule introduces new variables. Applying rules $R_{2}$ and $R_{3}$ makes the number of variables to decrease and $C^{\prime}=C \sigma$ where $\sigma=\operatorname{mgu}\left(t_{1}, t_{2}\right), t_{1}, t_{2}$ being subterms of $C$. The size of $\sigma$ is polynomial in $\left|t_{1}\right|$ and $\left|t_{2}\right|$ thus polynomial in $|C|$. Since terms are represented by dags $\left|C^{\prime}\right| \leq|C|+|\sigma|$. Applying the other rules makes the size of the constraint system to decrease. Hence $n$ is polynomially bounded in the size of $C$.

\section{A.3 Completeness}

Let $T_{1} \subseteq T_{2} \subseteq \cdots \subseteq T_{n}$. We say that a proof $\pi$ of $T_{i} \vdash u$ is left minimal if, for any $j<i$ such that $T_{j} \vdash u, \pi$ is also a proof of $T_{j} \vdash u$. We say that a proof is simple if any subproof is left minimal and on any branch there are no two equal nodes.

Lemma 5. If $T_{i} \vdash u$ then there is a simple proof of it. 
Proof. We prove the property by induction on the pair $(i, m)$ (considering again the lexicographical order), where $m$ is the size of a proof of $T_{i} \vdash u$.

If $i=1$ then the proof is left minimal and it exists a proof without repeating nodes on branches.

If $i>1$ and there is $j<i$ such that $T_{j} \vdash u$ then apply the recursion hypothesis to obtain the existence of a simple proof of $T_{j} \vdash u$. This proof is also a simple proof of $T_{i} \vdash u$.

If $i>1$ and there is no $j<i$ such that $T_{j} \vdash u$, then apply the recursion hypothesis on the immediate subproofs of the proof of $T_{i} \vdash u$. If the node $T_{i} \vdash u$ appears in the subproofs then consider one of those (sub)proofs of $T_{i} \vdash u$. Otherwise apply the same last rule to obtain the root node $T_{i} \vdash u$. Anyhow, the obtained proof and all of its proper subproofs are left minimal by construction, and hence the obtained proof is simple.

For a constraint system $C$, we call a left hand side $T_{i}$ minimal unsolved if for all $T \Vdash u \in C$ such that $T \subsetneq T_{i}, u$ is a variable. In this case we denote $T_{i}^{\prime}=T_{i} \cup$ $\left\{x \mid T \Vdash x \in C, T \subsetneq T_{i}\right\}$.

Lemma 6. Let $C$ be a constraint system, $\theta$ a solution and $T_{i}$ a minimal unsolved left hand side of $C$. If there is a simple proof of $T_{i} \theta \vdash u$ having the last rule an axiom or a decomposition then there is $t \in S t\left(T_{i}\right), t$ not a variable, such that $t \theta=u$.

Proof. Consider a simple proof of $T_{i} \theta \vdash u$. We reason by induction on the depth of the proof. We can have that:

- The last rule is an axiom. Then $u \in T_{i} \theta$ and hence there is $t \in T_{i}$ (thus $t \in$ $\operatorname{St}\left(T_{i}\right)$ ) such that $t \theta=u$. If $t$ is a variable then it exists $i_{1}<i$ such that $T_{i_{1}}=$ $\min \left\{T^{\prime} \mid T^{\prime} \Vdash u^{\prime} \in C, x \in \mathcal{V}\left(u^{\prime}\right)\right\}$ (see the definition of a constraint system). Since the proof of $T_{i} \theta \vdash u$ is left minimal, it is also a proof of $T_{i_{1}} \theta \vdash t \theta$, that is $t \theta \in T_{i_{1}} \theta$. Hence $t \theta=t_{1} \theta$, for some $t_{1} \in T_{i_{1}}$. If $t_{1}$ is a variable, we can continue in the same way, until we find a term $t_{k} \in T_{i_{k}}$ with $i_{k}<i$ such that $t_{k}$ is not a variable and $t_{k} \theta=u$. We eventually find such a term since $T_{1}$ has no variables. Since $T_{i_{k}} \subseteq T_{i}$ we have, as required, $t_{k} \in S t\left(T_{i}\right)$.

- The last rule is a decomposition.

Suppose that it is a symmetric decryption. That is, there is $w$ such that $T_{i} \theta \vdash$ enc $(u, w), T_{i} \theta \vdash w$. By simplicity of the proof, the last rule applied when obtaining enc $(u, w)$ was an axiom or a decomposition, otherwise the same node would appear twice. Then applying the induction hypothesis we have that there is $t_{1} \in \operatorname{St}\left(T_{i}\right)$, $t_{1}$ not a variable, such that $t_{1} \theta=\operatorname{enc}(u, w)$. It follows that $t_{1}=\operatorname{enc}\left(t_{1}^{\prime}, t_{1}^{\prime \prime}\right)$ with $t_{1}^{\prime} \theta=u$. If $t_{1}^{\prime}$ is a variable then $T_{t_{1}^{\prime}} \theta \vdash t_{1}^{\prime} \theta=u$. Since the proof is left minimal we have that $T_{t_{1}^{\prime}} \theta \vdash \operatorname{enc}(u, w)$. By induction hypothesis there is $t_{2} \in \operatorname{St}\left(T_{t_{1}^{\prime}}\right)$, $t_{2}$ not a variable such that $t_{2} \theta=\operatorname{enc}(u, w)$. It follows that $t_{2}=\operatorname{enc}\left(t_{2}^{\prime}, t_{2}^{\prime \prime}\right)$ with $t_{2}^{\prime} \theta=u$. If $t_{2}^{\prime}$ is a variable we can continue in the same way. We will finally find $t_{k}^{\prime} \in S t\left(T_{t_{k-1}^{\prime}}\right), t_{k}^{\prime}$ not a variable such that $t_{k}^{\prime} \theta=u$, because $T_{1}$ has no variables. For the other decomposition rules the same reasoning holds.

Lemma 7. Let $C$ be a constraint system, $\theta$ a solution and $T_{i}$ a minimal unsolved left hand side of $C$, such that $T_{i}$ does not contain two distinct non variable unifiable subterms. If $u \in S t\left(T_{i}\right), u$ non variable, and there is a simple proof of $u \theta$ from $T_{i} \theta$ then we have that $T_{i}^{\prime} \vdash u$, where $T_{i}^{\prime}=T_{i} \cup\left\{x \mid T \Vdash x \in C, T \subsetneq T_{i}\right\}$. 
Proof. We reason by induction on the depth of the proof. Again, according to the last applied rule in the simple proof of $T_{i} \theta \vdash u_{i} \theta$, we can have:

- The last rule is an axiom. Then $u \theta \in T_{i} \theta$. If $u \in T_{i}$ then $T_{i} \vdash u_{i}$ and hence $T_{i}^{\prime} \vdash u$. Otherwise, there is $t_{1} \in T_{i}$ such that $t_{1} \theta=u \theta$. We have $t_{1} \neq u$, and hence $t_{1}$ is a variable, since otherwise there is a contradiction with the hypothesis (there are two distinct non variable unifiable terms $u$ and $t_{1}$ in $S t\left(T_{i}\right)$ ). We then have $T_{t_{1}} \theta \vdash t_{1} \theta=u \theta$, and, by simplicity of the proof $u \theta \in T_{t_{1}} \theta$. There is then $t_{2} \in T_{t_{1}}$ such that $t_{2} \theta=u \theta$. As before $t_{2}$ is a variable. We can continue in this way, and we arrive at a contradiction since $T_{1}$ has no variables. Hence $u \in T_{i}$.

- The last rule is a decomposition.

Suppose for example that it is the symmetric decryption rule. That is, there is $w$ such that $T_{i} \theta \vdash \operatorname{enc}(u \theta, w), T_{i} \theta \vdash w$. Applying Lemma 6 we obtain that there is $t_{1} \in S t\left(T_{i}\right), t_{1}$ not a variable, such that $t_{1} \theta=\operatorname{enc}(u \theta, w)$. Since $t_{1}$ is not a variable we have that $t_{1}=\operatorname{enc}\left(t_{1}^{\prime}, t_{1}^{\prime \prime}\right)$. We have that $t_{1}^{\prime} \theta=u \theta$ and $t_{1}^{\prime \prime} \theta=w$. If $t_{1}^{\prime}$ is a variable then $T_{t_{1}^{\prime}} \theta \vdash t_{1}^{\prime} \theta$. Since the proof is left minimal we have that $T_{t_{1}^{\prime}} \theta \vdash \operatorname{enc}(u \theta, w)$. Then there is $t_{2} \in S t\left(T_{t_{1}^{\prime}}\right), t_{2}$ not a variable such that $t_{2} \theta=$ enc $(u \theta, w)$. It follows that $t_{2}=\operatorname{enc}\left(t_{2}^{\prime}, t_{2}^{\prime \prime}\right)$ with $t_{2}^{\prime} \theta=u \theta$ and $t_{2}^{\prime \prime} \theta=w$. If $t_{2}^{\prime}$ is a variable we can continue in the same way. Hence there is $t_{k} \in \operatorname{St}\left(T_{t_{k-1}^{\prime}}\right)$ (thus $\left.t_{k} \in S t\left(T_{i}\right)\right), t_{k}=\operatorname{enc}\left(t_{k}^{\prime}, t_{k}^{\prime \prime}\right)$, such that $t_{k}^{\prime}$ not a variable, $t_{k}^{\prime} \theta=u \theta$ and $t_{k}^{\prime \prime} \theta=w$. Since $t_{k}^{\prime}$ is not a variable, we have that $t_{k}^{\prime}=u$ (otherwise we would have two distinct non variable unifiable terms $t_{k}^{\prime}$ and $u$ in $S t\left(T_{i}\right)$ ). We can apply the induction hypothesis to obtain that $T_{i}^{\prime} \vdash \operatorname{enc}\left(t_{k}^{\prime}, t_{k}^{\prime \prime}\right)$. Now, if $t_{k}^{\prime \prime}$ is a variable then $t_{k}^{\prime \prime} \in T_{i}^{\prime}$, thus $T_{i}^{\prime} \vdash t_{k}^{\prime \prime}$. Otherwise, if $t_{k}^{\prime \prime}$ is not a variable then, by induction hypothesis, $T_{i}^{\prime} \vdash t_{k}^{\prime \prime}$. Hence, in both cases, we obtain that $T_{i}^{\prime} \vdash u$.

For the other decomposition rules the same reasoning holds.

- The last rule is a composition.

Suppose for example that it is the symmetric encryption rule. Then $u \theta=\operatorname{enc}\left(w_{1}, w_{2}\right)$ and $T_{i} \theta \vdash w_{1}$ and $T_{i} \theta \vdash w_{2}$. Since $u$ is not a variable we have that $u=\operatorname{enc}\left(u_{1}, u_{2}\right)$, $u_{1} \theta=w_{1}$ and $u_{2} \theta=w_{2}$. If $u_{1}$ (resp. $u_{2}$ ) is a variable then $u_{1}$ (resp. $u_{2}$ ) is in $T_{i}^{\prime}$ (this is because $u \in S t\left(T_{i}\right)$ ). Otherwise we apply the recursion hypothesis. Hence in both cases we have $T_{i}^{\prime} \vdash u_{1}$ and $T_{i}^{\prime} \vdash u_{2}$. Thus $T_{i}^{\prime} \vdash u$.

For the other composition rules the same reasoning holds.

Lemma 8 (completeness). If $C$ is an unsolved constraint system and $\theta$ is a solution of $C$ for the property $P$ w.r.t. $L$ then there is a constraint system $C^{\prime}$ and solution $\tau$ of $C^{\prime}$ for the property $P$ w.r.t. $L \sigma$ such that $C \rightsquigarrow_{\sigma} C^{\prime}$ and $\theta=\sigma \tau$.

Proof. Consider the minimal unsolved constraint $T_{i} \Vdash u_{i}, u_{i}$ is not a variable and $u_{j}$ is a variable, for all $j<i$.

We have $T_{i} \theta \vdash u_{i} \theta$. Consider a simple proof of $T_{i} \theta \vdash u_{i} \theta$. According to the last applied rule in this proof, we can have:

1. The last rule is a composition.

Suppose that it is the pairing rule. That is, there are $w_{1}, w_{2}$ such that $T_{i} \theta \vdash w_{1}$, $T_{i} \theta \vdash w_{2}$ and $\left\langle w_{1}, w_{2}\right\rangle=u_{i} \theta$. Since $u_{i}$ is not a variable there exists $u^{\prime}, u^{\prime \prime}$ such that $u_{i}=\left\langle u^{\prime}, u^{\prime \prime}\right\rangle$. Hence we can apply the simplification rule $R_{\langle\rangle}$in order to 
obtain $C^{\prime}$. Since $u^{\prime} \theta=w_{1}$ and $u^{\prime \prime} \theta=w_{2}$, the substitution $\theta$ is also a solution to $C^{\prime}$ for $P$ w.r.t $L$.

For the other composition rules the same reasoning holds, applying this time the corresponding $R_{f}$ rule.

2. The last rule is an axiom or a decomposition. Applying Lemma 6 we obtain that there is $t \in S t\left(T_{i}\right), t$ not a variable, such that $t \theta=u_{i} \theta$. We can have the following two possibilities:

(a) If $t \neq u_{i}$ then we apply the simplification rule $R_{2}$.

(b) Otherwise, if $t=u_{i}$, then $u \in S t\left(T_{i}\right)$. We consider the cases:

i. There are two distinct non variable unifiable terms $t_{1}, t_{2} \in S t(T)$. Then we apply the simplification rule $R_{3}$.

ii. Otherwise, the simplification rule $R_{1}$ can be applied. This follows from Lemma 7.

\section{B Decidability of key cycles}

This section is devoted to the proof Lemma 1. We first introduce an intermediate lemma.

Lemma 9. Let $T \Vdash x$ be a constraint of a solved constraint system $C$ and $\theta$ be a simple solution. Let $m, u, k$ be terms such that

$$
T \theta \vdash m \text { and } \operatorname{enc}(u, k) \sqsubseteq m \text { and } T \theta \forall k \text {. }
$$

Then there exists a non variable term $w$ such that $w \sqsubseteq w^{\prime}$ for some $w^{\prime} \in T$ and $w \theta=\operatorname{enc}(u, k)$.

Consider the index $i$ of the constraint $T \Vdash x$, that is such that $T_{i} \Vdash u_{i} \in C, T_{i}=T$ and $u_{i}=x$. The lemma is proved by induction on $(i, n)$ (lexicographical order) where $n$ is the length of the proof of $T_{i} \theta \vdash m$. Consider the last rule of the proof:

- (axiom rule) $m=t \theta$ for some $t \in T_{i}$. We can have that either there is $t^{\prime} \sqsubseteq t$ such that $t^{\prime} \theta=\operatorname{enc}(u, k)$, either enc $(u, k) \sqsubseteq y \theta$ for some $y \in \mathcal{V}(t)$. In the first case take $w=t^{\prime}$. In the second case, by definition of constraint systems, there exists $T_{j} \Vdash y \in C$ with $j<i$. Since $T_{j} \theta \vdash m$ and $T_{j} \theta \not k$ (since $T_{j} \subseteq T_{i}$ ), we deduce by induction hypothesis that there exists a non variable term $w$ such that $w \sqsubseteq w^{\prime}$ for some $w^{\prime} \in T_{i}$ and $w \theta=\operatorname{enc}(u, k)$.

- (decomposition rule) Let $m^{\prime}$ be the premise of the rule. We have that $T_{i} \theta \vdash m^{\prime}$ (with a proof of a strictly smaller length) and $m \sqsubseteq m^{\prime}$ thus enc $(u, k) \sqsubseteq m^{\prime}$. By induction hypothesis, we deduce that there exists a non variable term $w$ such that $w \sqsubseteq w^{\prime}$ for some $w^{\prime} \in T_{i}$ and $w \theta=\operatorname{enc}(u, k)$.

- (composition rule) All cases are similar to the previous one except if $m=\operatorname{enc}(u, k)$ and the rule is $\frac{S \vdash x \quad S \vdash y}{S \vdash \operatorname{enc}(x, y)}$. But this case contradicts $T_{i} \theta \forall k$.

We are now ready to prove Lemma 1. 
Lemma 1. Let $\theta$ be a partial solution of $C$ and $k, k^{\prime} \in K$ be two non deducible keys, that is lhs $(C) \theta \forall k, k^{\prime}$. If $k \rho_{e}^{L_{s} \theta} k^{\prime}$, that is $k$ encrypts $k^{\prime}$ in $L_{s} \theta$ then $\left(k, k^{\prime}\right) \in A$. Conversely, if $\left(k, k^{\prime}\right) \in A$ then $k \rho_{e}^{L_{s} \tau} k^{\prime}$, that is $k$ encrypts $k^{\prime}$ in $L_{s} \tau$.

Let us first remark there is no $x \in \mathcal{V}(C)$ such that $x \theta=k$ (or $k^{\prime}$ ), otherwise we would have that there exists $T \Vdash x \in C$ such that $T \theta \vdash x \theta=k$ i.e. $k$ is deducible from $L_{s} \theta$ (since $l h s(C) \subseteq L_{s}$ ).

Suppose that $k$ encrypts $k^{\prime}$ in $L_{s} \theta$. for some partial solution $\theta$. This implies that there are terms $u, v$ such that $v \in L_{s} \theta, \operatorname{enc}(u, k) \sqsubseteq v$ and $k^{\prime} \rho_{1} u$. We can have that either (first case) there are $w, w^{\prime}$ such that $w \sqsubseteq w^{\prime} \in L_{s}, w$ non variable and enc $(u, k)=w \theta$, either (second case) enc $(u, k) \sqsubseteq x \theta$ with $x \in \mathcal{V}\left(L_{s}\right)$. In the second case, consider the constraint $T_{x} \Vdash x \in C$. We have $T_{x} \theta \vdash x \theta$. Hence we can apply Lemma 9 for $x \theta$, $u$ and $k$ to obtain that there exists a non variable term $w$ such that $w \sqsubseteq w^{\prime} \in T_{x}$ and $w \theta=\operatorname{enc}(u, k)$. Hence, in both cases, we obtain that there are $w, w^{\prime}, w \sqsubseteq w^{\prime} \in L_{s} \cup T_{L}$ (since $\left.T_{x} \subseteq T_{L}\right)$ such that $w=\operatorname{enc}\left(w_{0}, k\right)$ (since $k$ is not deducible).

We have $w_{0} \theta=u$. Since $k^{\prime} \rho_{1} u$ and $k^{\prime}$ is a name or a variable, we can have that $k^{\prime} \rho_{1} w_{0}$ or $k^{\prime} \rho_{1} y \theta$ for some $y \in \mathcal{V}\left(w_{0}\right)$. If $k^{\prime} \rho_{1} w_{0}$ then $k$ encrypts $k^{\prime}$ in $L_{s} \cup T_{L}$. Hence, by construction of the graph $G$, we have that $\left(k, k^{\prime}\right) \in A$. If $k^{\prime} \rho_{1} y \theta$ then $k^{\prime} \rho_{1} y \tau$ since $k^{\prime}$ is not deducible, $\theta$ cannot introduce new keys and $\tau$ contains all the available keys. Hence, again by construction of the graph $G$, we have that $\left(k, k^{\prime}\right) \in A$.

Conversely, suppose that $\left(k, k^{\prime}\right)$ is an arc in the graph $G$. Then, from the construction of the graph, we can have the following possibilities. Either $k$ encrypts $k^{\prime}$ in $L_{s} \cup T_{L}$ or $k$ encrypts $x$ in $L_{s} \cup T_{L}$ and $k^{\prime} \rho_{1} x \tau$ for some variable $x \in \mathcal{V}\left(L_{s} \cup T_{L}\right)$. If $k$ encrypts $k^{\prime}$ in $L_{s}$ then $k$ encrypts $k^{\prime}$ in $L_{s} \tau$. If $k$ encrypts $x$ in $L_{s}$ and $k^{\prime} \rho_{1} x \tau$ then $k$ encrypts $k^{\prime}$ in $L_{s} \tau$. If $k$ encrypts $k^{\prime}$ in $T_{L}$ then $k$ encrypts $k^{\prime}$ in $t_{y}$, where $T_{y}=T_{L}, y \in \mathcal{V}\left(L_{s}\right)$. Hence $k$ encrypts $k^{\prime}$ in $y \tau$, thus in $L_{s} \tau$. Finally, if $k$ encrypts $x$ in $T_{L}$ and $k^{\prime} \rho_{1} x \tau$ then $k$ encrypts $k^{\prime}$ in $T_{L} \tau$, hence as before, in $t_{y}$ and thus in $L_{s} \tau$. Therefore in all cases we have that $k$ encrypts $k^{\prime}$ in $L_{s} \tau$. 